\title{
Hydrochemistry and nutrients of Bitter and Temsah Lakes, Suez Canal, Egypt.
}

Mohamed A. Hamed, Mohamed A. El-Sawy and Emad H. Abu El-Naga

National Institute of Oceanography and Fisheries, Suez and Aqaba Gulfs Branch, Egypt.

\section{ABSTRACT}

Temperature, pH, salinity, Dissolved Oxygen (DO), Biological Oxygen Demand (BOD) and Oxidizable Organic Matter (OOM) as well as ammonia, nitrite, nitrate, phosphate and silicate were studied seasonally in the surface water of Bitter and Temsah Lakes during 2009 and 2010.

The results revealed that: i) the annual mean range of concentration of these parameters in Bitter lakes were $\left(24.53^{\circ} \mathrm{C} ; 8.09 ; 40.40 \%\right.$; $7.26 \mathrm{mgO}_{2} / \mathrm{L} ; 1.74 \mathrm{mgO}_{2} / \mathrm{L}$; $1.29 \mathrm{mgO}_{2} / \mathrm{L} ; 1.69 \mu \mathrm{mol} / \mathrm{L} ; 0.52 \mu \mathrm{mol} / \mathrm{L} ; 2.91 \mu \mathrm{mol} / \mathrm{L} ; 0.62 \mu \mathrm{mol} / \mathrm{L}$ and 3.13 $\mu \mathrm{mol} / \mathrm{L}$, respectively). On the other hand, the corresponding values in Temsah Lake were $\left(25.07^{\circ} \mathrm{C} ; 8.11 ; 28.84 \%\right.$ \% $6.96 \mathrm{mgO}_{2} / \mathrm{L} ; 3.35 \mathrm{mgO}_{2} / \mathrm{L} ; 2.70 \mathrm{mgO}_{2} / \mathrm{L} ; 2.78$ $\mu \mathrm{mol} / \mathrm{L} ; 0.92 \mu \mathrm{mol} / \mathrm{L} ; 6.16 \mu \mathrm{mol} / \mathrm{L} ; 1.03 \mu \mathrm{mol} / \mathrm{L}$; and $4.99 \mu \mathrm{mol} / \mathrm{L}$, respectively).

ii) The aquatic system of Temsah Lake became deteriolized, where the concentration of contaminants viz: BOD, OOM, and nutrient salts were relatively increased, probably due to the effect of acute pollution of the disposal of untreated sewage and agriculture effluents from sweet Canal.

iii) Seasonally, nutrient salts recorded the highest values during winter and this is may be due to tidal current of the water column, which mixes the bottom water with the surface. While the lowest values were recorded during autumn and spring, due to the production of phytoplankton standing crop

iv) The calculated N: P: Si ratios are less than that of Redfield ratio, suggesting that the areas under investigation (Bitter and Temsah Lakes) are $\mathrm{N}$ limited, and v).

The level of nutrients and other trophic indicating parameters, refer that the areas of investigation are in eutrophic state.

Keywords: water quality, nutrients, Bitter Lakes, Temsah Lake, Suez Canal.

\section{INTRODUCTION}

Suez Canal is, actually, the lonely canal linking between the Mediterranean Sea and the Red Sea. It is located between Suez and Port Said provinces (lying between longitudes $32^{\circ} 20^{\prime}$ and $32^{\circ} 35^{\prime} \mathrm{E}$ and between latitudes $29^{\circ} 55^{\prime}$ and $31^{\circ} 15^{\prime} \mathrm{N}$ with an average length of $164 \mathrm{~km}$ along the major axis).

Due to the importance of the Suez Canal for navigation and global mind link between the Mediterranean and the Red Sea, the Bitter Lakes and Lake Temsah have been chosen for different environmental studies. Recent urbanization of the region has led to the situation that the Suez Canal system is suffering considerably from pollution, since it acts as a big navigation route.

Temsah Lake covers about $15 \mathrm{Km}^{2}$ between $30^{\circ} 32^{\prime}$ and $30^{\circ} 36^{\prime}$ north latitude and $32^{\circ} 16^{\prime}$ and $32^{\circ} 21^{\prime}$ east longitude and is located near the middle of the Suez Canal at a point $80 \mathrm{Km}$ south of Port Said. The depth of the lake ranges between 6 and $13 \mathrm{~m}$. Following the construction of High Dam (completed in 1970), lake water quality changed from saline to fresh water due to precipitation of gypsum and mud lamina. The lake is the backbone of a tourism industry that attracts a large number of the holiday visitors. In addition to the visitors, the tourism and fishing industries employ local citizens and provide a significant portion of the district revenues. 
On the other hand the Bitter Lakes $\left(30^{\circ} 20^{\prime} \mathrm{N}, 32^{\circ} 23^{\prime} \mathrm{E}\right)$ are the largest water bodies along the length of the Suez Canal, containing about $85 \%$ of the system's water. The Great and Small Bitter Lakes, separated by narrows, are saline lakes situated between the north and south parts of the Suez Canal (El-Bassat, 2008). The Bitter Lakes now have a surface area of about $250 \mathrm{~km}^{2}$. To the north, the canal also runs through Lake Manzala and Lake Temsah.

As the canal has no locks, sea water flows freely into the lakes from both the Mediterranean Sea and the Red Sea, replacing water lost due to evaporation. The lakes act as a buffer for the canal, reducing the effect of tidal currents (Touliabah and Taylor 2004). The Bitter Lakes suffer from various types of pollution, including domestic sewage from the surrounding human settlements, thermal pollution from Abu-Sultan electric power location's cooling water, as well as industrial and agricultural wastes from Ismailia City via the Malaria Drain, which also collects agricultural wastes from the cultivated lands on the west bank of the Great Bitter Lake (Badawy 2001).

Pollution in Bitter and Temsah Lakes has considerably increased in recent years, through oil ships, agricultural, industrial and domestic drainage. Consequently, the present work aims to investigate water quality and nutrient salt concentrations in surface water of the Bitter and Temsah lakes.

\section{MATERIALS AND METHODS}

\section{Sampling}

Surface water samples were collected seasonally during of 2009-2010 from nine locations adequately covering the different Lake's basin. Five locations were chosen in Bitter Lakes and four locations were in Temsah Lake (Fig. 1).

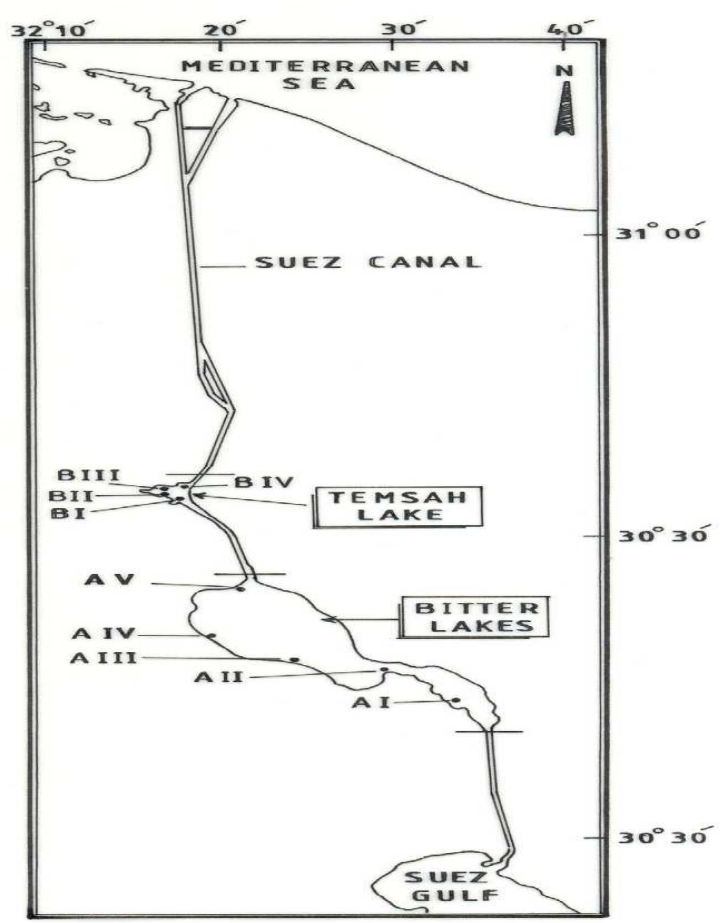

Fig. 1: Sampling stations of the investigated area along the Suez Canal during 2009-2010. A= Great Bitter Lakes $(A 1=$ Shandora, AII Kabrite, AIII = Fanara, AIV= Fayed and AV= Deversoir) and B=El-Temsah Lake (BI- BIV)

\section{Procedures}

Surface water temperature was measured at the time of sampling using standard (0-100 $\left.{ }^{\circ} \mathrm{C}\right)$ thermometer. Hydrogen ion concentration was measured at the time of sampling on the board of the ship using a pocket $\mathrm{pH}$ meter (Orion 210). Salinity was determined using an 
inductive salinometer (S.C.T. meters) from yellow springs Instrument Co. model 33. Dissolved oxygen was determined by the Winkler method (1988) taking all the necessary precautions into consideration. Biological Oxygen Demand (BOD) and Oxidizable Organic Matter (OOM) were measured according to standard methods, (APHA, 1995). The nutrients $\mathrm{NH}_{4}, \mathrm{NO}_{2}, \mathrm{NO}_{3}, \mathrm{PO}_{4}$ and silicate were measured in the filtered water samples (after filtration, using $0.45 \mu \mathrm{m}$ membrane filters), following the procedures of Strickland and Parsons (1975).

The correlation coefficient $(r)$ was calculated between the environmental parameters using statistics program SPSS version 18.

\section{RESULTS AND DISCUSSION}

\section{Environmental characteristics:}

The Suez Canal is considered among the warm temperature zone and merely as a gradient of decreasing temperatures from the Red Sea to the Mediterranean. Limited spatial variations in surface water temperature were measured; these variations depend primarily on the weather conditions and partly on the time of sampling.

In general, the seasonal temperature variations follow these of prevailing climate conditions. Temperature of surface water in Bitter Lake ranged between $16.90^{\circ} \mathrm{C}$ during winter at location 1 to $31.35^{\circ} \mathrm{C}$ during summer at location 4 . On the other hand, temperature in Temsah Lake ranged between $16.20^{\circ} \mathrm{C}$ during winter at location 9 to $32.50^{\circ} \mathrm{C}$ during summer at location 9. This may be due to several factors such as air temperature, wind, waves and gain or loss of heat in shallow waters close to the coast. The discharge of heated water from a thermal power plant raised the water temperature in the impacted area by $13^{\circ} \mathrm{C}$ in autumn and $22^{\circ} \mathrm{C}$ in summer and also caused decreases in chlorophyll and phytoplankton abundance (Ashour et al., 2000). From the above results, high temperatures occurred in summer, while low values were observed in winter.

The values of hydrogen ion concentration in the Bitter Lakes ranged between the lowest value (7.91) during autumn at location 4 (Table 1), probably due to the disposal of fresh water from drains into the Suez Canal and the highest values of $\mathrm{pH}(8.29)$ during winter at location 4 and $\mathrm{On}$ the other hand, $\mathrm{pH}$ in Temsah Lake varied between 7.78 during autumn at location 6 and 8.31 during summer at location 9. The relatively higher $\mathrm{pH}$ values were recorded during the warm seasons in which the rise in temperature usually stimulates phytoplankton photosynthetic activity causing more consumption of carbon dioxide and the rise of $\mathrm{pH}$ (Abdallah et al., 1995)

Table 1: Hydrographic parameters of Bitter and Temsah Lakes during Autumn 2009.

\begin{tabular}{|c|c|c|c|c|c|c|c|}
\hline & Locations & Temp. & $\mathrm{pH}$ & S\%o & DO & BOD & OOM \\
\hline \multirow{5}{*}{ 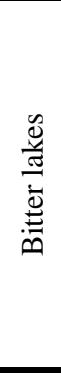 } & 1 & $\begin{array}{c}24.10-25.60 \\
(24.85)\end{array}$ & $\begin{array}{c}8.01-8.06 \\
(8.04) \\
\end{array}$ & $\begin{array}{c}40.20-40.60 \\
(40.43) \\
\end{array}$ & $\begin{array}{c}5.95-6.76 \\
(6.36) \\
\end{array}$ & $\begin{array}{c}1.21-1.87 \\
(1.48) \\
\end{array}$ & $\begin{array}{c}0.88-0.92 \\
(0.83) \\
\end{array}$ \\
\hline & 2 & $\begin{array}{c}24.10-27.10 \\
(25.60)\end{array}$ & $\begin{array}{c}8.01-8.05 \\
(8.03) \\
\end{array}$ & $\begin{array}{c}40.40-41.20 \\
(40.70)\end{array}$ & $\begin{array}{c}6.24-6.56 \\
(6.40) \\
\end{array}$ & $\begin{array}{c}1.48-2.82 \\
(2.08) \\
\end{array}$ & $\begin{array}{c}0.72-1.20 \\
(0.93) \\
\end{array}$ \\
\hline & 3 & $\begin{array}{c}26.30-30.00 \\
(28.15)\end{array}$ & $\begin{array}{c}8.04-8.12 \\
(8.09)\end{array}$ & $\begin{array}{c}39.30-40.35 \\
(40.06)\end{array}$ & $\begin{array}{c}6.45-7.28 \\
(6.87)\end{array}$ & $\begin{array}{c}1.35-1.67 \\
(1.56)\end{array}$ & $\begin{array}{c}1.08-1.44 \\
(1.21)\end{array}$ \\
\hline & 4 & $\begin{array}{c}26.00-30.60 \\
(28.30)\end{array}$ & $\begin{array}{c}7.91-8.04 \\
(7.98)\end{array}$ & $\begin{array}{c}39.70-40.30 \\
(39.60)\end{array}$ & $\begin{array}{c}6.81-7.69 \\
(7.25) \\
\end{array}$ & $\begin{array}{c}1.68-2.09 \\
(1.86)\end{array}$ & $\begin{array}{c}1.26-1.93 \\
(1.50) \\
\end{array}$ \\
\hline & 5 & $\begin{array}{c}25.10-28.50 \\
(26.80)\end{array}$ & $\begin{array}{c}8.06-8.06 \\
(8.04)\end{array}$ & $\begin{array}{c}39.70-40.30 \\
(39.96) \\
\end{array}$ & $\begin{array}{c}6.76-7.05 \\
(6.91) \\
\end{array}$ & $\begin{array}{c}1.52-1.75 \\
(1.64)\end{array}$ & $\begin{array}{c}1.26-1.72 \\
(1.46)\end{array}$ \\
\hline \multirow{4}{*}{ 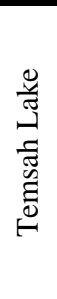 } & 6 & $\begin{array}{c}24.50-28.40 \\
(26.45)\end{array}$ & $\begin{array}{c}7.78-7.98 \\
(7.88)\end{array}$ & $\begin{array}{c}24.70-32.10 \\
(28.40)\end{array}$ & $\begin{array}{c}5.44-6.14 \\
(5.83)\end{array}$ & $\begin{array}{c}4.38-5.85 \\
(5.27)\end{array}$ & $\begin{array}{c}3.28-4.16 \\
(3.66)\end{array}$ \\
\hline & 7 & $\begin{array}{c}24.70-29.60 \\
(27.15)\end{array}$ & $\begin{array}{c}8.06-8.21 \\
(8.14)\end{array}$ & $\begin{array}{c}28.90-39.10 \\
(34.30)\end{array}$ & $\begin{array}{c}5.86-6.46 \\
(6.11)\end{array}$ & $\begin{array}{c}2.96-3.76 \\
(3.66)\end{array}$ & $\begin{array}{c}2.40-3.29 \\
(2.85)\end{array}$ \\
\hline & 8 & $\begin{array}{c}25.70-29.00 \\
(27.35)\end{array}$ & $\begin{array}{c}8.24-8.29 \\
(8.27)\end{array}$ & $\begin{array}{c}30.20-30.70 \\
(30.45)\end{array}$ & $\begin{array}{c}5.83-6.58 \\
(6.20)\end{array}$ & $\begin{array}{c}2.12-2.96 \\
(2.46)\end{array}$ & $\begin{array}{c}1.62-2.03 \\
(2.32)\end{array}$ \\
\hline & 9 & $\begin{array}{c}25.10-29.50 \\
(27.30)\end{array}$ & $\begin{array}{c}8.14-8.21 \\
(8.27)\end{array}$ & $\begin{array}{c}29.50-36.20 \\
(32.56)\end{array}$ & $\begin{array}{c}\text { 6.18-6.71 } \\
(6.43)\end{array}$ & $\begin{array}{c}2.45-4.18 \\
(2.83)\end{array}$ & $\begin{array}{c}2.25-2.61 \\
(2.47) \\
\end{array}$ \\
\hline
\end{tabular}


The results indicated that, the salinity of the surface water reached its maximum during summer and early autumn and the minimum in winter. The relatively high increasing in the salinity during summer may be due to the increasing of the evaporation rate of water, shallowness of lake water and high temperature during this season (Morcos, 1970). The water salinity in the Suez Canal showed wide fluctuations between locations especially in those near fresh water outlets. Thus, in Bitter lakes the highest salinity $(41.60 \%$ ) was found during summer at location 2 (Table 2), while the lowest value (39.20\%o) was found during winter at location 5 (Table 3). The highest salinity was concentrated at the Bitter Lakes, due to the high rate of evaporation in the region; and partly due to dissolution of evaporate deposits at the bottom of the Lakes (Morcos and Riley, 1966).

Table 1: Hydrographic parameters of Bitter and Temsah Lakes during summer 2009.

\begin{tabular}{|c|c|c|c|c|c|c|c|}
\hline & Locations & Temp. & $\mathrm{pH}$ & $\mathrm{S} \%$ & DO & BOD & OOM. \\
\hline \multirow{5}{*}{ 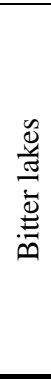 } & 1 & $\begin{array}{c}28.75-29.20 \\
(28.97)\end{array}$ & $\begin{array}{c}7.95-8.01 \\
(7.85)\end{array}$ & $\begin{array}{c}40.50-41.35 \\
(40.92)\end{array}$ & $\begin{array}{c}5.70-6.78 \\
(6.24)\end{array}$ & $\begin{array}{c}1.74-2.27 \\
(1.92)\end{array}$ & $\begin{array}{c}1.99-2.07 \\
(2.05)\end{array}$ \\
\hline & 2 & $\begin{array}{c}29.00-29.50 \\
(29.25)\end{array}$ & $\begin{array}{c}7.97-8.05 \\
(8.01)\end{array}$ & $\begin{array}{c}40.70-41.60 \\
(41.15)\end{array}$ & $\begin{array}{c}6.08-7.70 \\
(6.89)\end{array}$ & $\begin{array}{c}1.19-2.12 \\
(1.63)\end{array}$ & $\begin{array}{c}0.98-1.75 \\
(1.35)\end{array}$ \\
\hline & 3 & $\begin{array}{c}30.25-31.25 \\
(30.75)\end{array}$ & $\begin{array}{c}7.99-8.02 \\
(8.01)\end{array}$ & $\begin{array}{c}40.30-40.55 \\
(40.42)\end{array}$ & $\begin{array}{c}6.10-6.59 \\
(6.35)\end{array}$ & $\begin{array}{c}1.48-1.82 \\
(1.68)\end{array}$ & $\begin{array}{c}0.96-1.54 \\
(1.23)\end{array}$ \\
\hline & 4 & $\begin{array}{c}30.75-31.35 \\
(31.05)\end{array}$ & $\begin{array}{c}8.03-8.11 \\
(8.07)\end{array}$ & $\begin{array}{c}40.01-40.15 \\
(40.07)\end{array}$ & $\begin{array}{c}6.48-7.02 \\
(6.75)\end{array}$ & $\begin{array}{c}1.69-2.59 \\
(2.13)\end{array}$ & $\begin{array}{c}1.60-2.16 \\
(1.36)\end{array}$ \\
\hline & 5 & $\begin{array}{c}30.50-31.00 \\
(30.75)\end{array}$ & $\begin{array}{c}7.96-8.06 \\
(8.02) \\
\end{array}$ & $\begin{array}{c}39.90-39.98 \\
(39.94)\end{array}$ & $\begin{array}{c}7.04-7.39 \\
(7.17) \\
\end{array}$ & $\begin{array}{c}1.80-1.98 \\
(1.86)\end{array}$ & $\begin{array}{c}1.25-1.96 \\
(1.59) \\
\end{array}$ \\
\hline \multirow{4}{*}{ 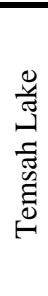 } & 6 & $\begin{array}{c}29.50-30.80 \\
(30.15)\end{array}$ & $\begin{array}{c}7.89-7.99 \\
(7.94)\end{array}$ & $\begin{array}{c}30.60-38.00 \\
(31.13)\end{array}$ & $\begin{array}{c}5.20-5.78 \\
(5.33)\end{array}$ & $\begin{array}{c}4.38-5.92 \\
(5.13)\end{array}$ & $\begin{array}{c}3.76-4.24 \\
(3.96)\end{array}$ \\
\hline & 7 & $\begin{array}{c}31.00-31.50 \\
(31.25)\end{array}$ & $\begin{array}{c}7.96-8.17 \\
(8.06)\end{array}$ & $\begin{array}{c}33.90-40.10 \\
(36.56)\end{array}$ & $\begin{array}{c}6.10-6.34 \\
(6.18)\end{array}$ & $\begin{array}{c}3.54-4.79 \\
(4.26)\end{array}$ & $\begin{array}{c}2.38-2.85 \\
(2.59)\end{array}$ \\
\hline & 8 & $\begin{array}{c}31.20-32.00 \\
(31.60)\end{array}$ & $\begin{array}{c}8.11-8.16 \\
(8.13)\end{array}$ & $\begin{array}{c}32.00-36.80 \\
(34.40)\end{array}$ & $\begin{array}{c}6.48-7.23 \\
(6.81)\end{array}$ & $\begin{array}{c}2.25-3.16 \\
(2.62)\end{array}$ & $\begin{array}{c}2.09-2.84 \\
(2.47)\end{array}$ \\
\hline & 9 & $\begin{array}{c}31.50-32.50 \\
(32.00)\end{array}$ & $\begin{array}{c}8.13-8.31 \\
(8.22)\end{array}$ & $\begin{array}{c}33.80-37.30 \\
(35.73)\end{array}$ & $\begin{array}{c}6.26-7.48 \\
(6.76)\end{array}$ & $\begin{array}{c}1.89-3.41 \\
(2.76)\end{array}$ & $\begin{array}{c}1.91-3.35 \\
(1.61)\end{array}$ \\
\hline
\end{tabular}

Table 3: Hydrographic parameters of Bitter and Temsah Lakes during winter 2010.

\begin{tabular}{|c|c|c|c|c|c|c|c|}
\hline & Locations & Temp. & $\mathrm{pH}$ & $\mathrm{S} \% \mathrm{o}$ & DO & BOD & OOM. \\
\hline \multirow{5}{*}{ 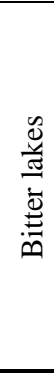 } & 1 & $\begin{array}{c}16.90-18.50 \\
(17.61)\end{array}$ & $\begin{array}{c}8.06-8.18 \\
(8.13)\end{array}$ & $\begin{array}{c}40.10-40.40 \\
(40.26)\end{array}$ & $\begin{array}{c}6.30-9.12 \\
(7.71)\end{array}$ & $\begin{array}{c}1.42-1.66 \\
(1.54)\end{array}$ & $\begin{array}{c}1.39-1.77 \\
(1.53)\end{array}$ \\
\hline & 2 & $\begin{array}{c}18.00-19.00 \\
(18.60)\end{array}$ & $\begin{array}{c}8.10-8.18 \\
(8.16)\end{array}$ & $\begin{array}{c}40.10-40.60 \\
\quad(40.40)\end{array}$ & $\begin{array}{c}6.60-8.20 \\
(7.40)\end{array}$ & $\begin{array}{c}1.67-1.75 \\
(1.71)\end{array}$ & $\begin{array}{c}0.98-1.35 \\
(1.13)\end{array}$ \\
\hline & 3 & $\begin{array}{c}18.10-19.25 \\
(18.85)\end{array}$ & $\begin{array}{c}8.05-8.21 \\
(8.10)\end{array}$ & $\begin{array}{c}40.30-40.90 \\
(40.56)\end{array}$ & $\begin{array}{c}6.55-8.45 \\
(7.50)\end{array}$ & $\begin{array}{c}1.28-1.72 \\
(1.50)\end{array}$ & $\begin{array}{c}0.76-1.44 \\
(1.03)\end{array}$ \\
\hline & 4 & $\begin{array}{c}18.00-19.20 \\
(18.46)\end{array}$ & $\begin{array}{c}8.05-8.29 \\
(8.20)\end{array}$ & $\begin{array}{c}39.70-40.10 \\
(39.83)\end{array}$ & $\begin{array}{c}6.97-7.78 \\
(7.38) \\
\end{array}$ & $\begin{array}{c}1.88-2.15 \\
(2.01)\end{array}$ & $\begin{array}{c}1.30-2.06 \\
(1.70)\end{array}$ \\
\hline & 5 & $\begin{array}{c}17.50-19.00 \\
(18.43)\end{array}$ & $\begin{array}{c}8.18-8.26 \\
(8.26)\end{array}$ & $\begin{array}{c}39.20-40.90 \\
(39.93)\end{array}$ & $\begin{array}{c}6.86-7.18 \\
(7.02)\end{array}$ & $\begin{array}{c}1.61-1.96 \\
(1.78)\end{array}$ & $\begin{array}{c}1.05-1.66 \\
(1.20)\end{array}$ \\
\hline \multirow{4}{*}{$\begin{array}{l}\text { है } \\
\text { ज्ञ } \\
\text { हే } \\
\text { है } \\
\text { है }\end{array}$} & 6 & $\begin{array}{c}17.20-19.80 \\
(18.50)\end{array}$ & $\begin{array}{c}7.86-8.12 \\
(8.00)\end{array}$ & $\begin{array}{c}7.30-39.10 \\
(23.20)\end{array}$ & $\begin{array}{c}5.87-6.12 \\
(5.99)\end{array}$ & $\begin{array}{c}3.41-3.88 \\
(3.64)\end{array}$ & $\begin{array}{c}3.46-4.14 \\
(3.80)\end{array}$ \\
\hline & 7 & $\begin{array}{c}17.00-19.40 \\
(18.26)\end{array}$ & $\begin{array}{c}8.00-8.23 \\
(8.09)\end{array}$ & $\begin{array}{c}12.70-24.50 \\
(16.93)\end{array}$ & $\begin{array}{c}6.73-7.33 \\
(7.05)\end{array}$ & $\begin{array}{c}2.75-3.15 \\
(2.93)\end{array}$ & $\begin{array}{c}2.18-2.65 \\
(2.50)\end{array}$ \\
\hline & 8 & $\begin{array}{c}16.50-19.60 \\
(18.20)\end{array}$ & $\begin{array}{c}8.02-8.28 \\
(8.14) \\
\end{array}$ & $\begin{array}{c}10.01-23.20 \\
(16.60)\end{array}$ & $\begin{array}{c}6.20-6.73 \\
(6.80) \\
\end{array}$ & $\begin{array}{c}2.12-2.46 \\
(2.29) \\
\end{array}$ & $\begin{array}{c}1.99-2.44 \\
(2.16) \\
\end{array}$ \\
\hline & 9 & $\begin{array}{c}16.20-19.50 \\
(18.06)\end{array}$ & $\begin{array}{c}8.01-8.22 \\
(8.16)\end{array}$ & $\begin{array}{c}8.80-22.90 \\
(15.85)\end{array}$ & $\begin{array}{c}6.15-6.44 \\
(6.26)\end{array}$ & $\begin{array}{c}2.33-2.56 \\
(2.44)\end{array}$ & $\begin{array}{c}1.71-3.15 \\
(1.81)\end{array}$ \\
\hline
\end{tabular}

On the other hand the variation of salinity in Temsah Lake was great because of the presence of freshwater sources; the maximum value of salinity in Temsah Lake $(40.10 \%$ ) was recorded during summer at location 7 (Table 1), while the minimum value was found $(7.30 \%$ ) at location 6 during winter (Table 3 ) and this may be due to 
direct effect by Ismailia sweet Canal and western lagoon receiving the sewage discharge and waste waters.

The annual mean and seasonal variations of dissolved oxygen in water samples from Bitter and Temsah Lakes are given in Table 5. Regionally, dissolved oxygen in Bitter Lakes fluctuated between $5.70 \mathrm{mgO}_{2} / \mathrm{L}$ at location 1 and $10.55 \mathrm{mgO}_{2} / \mathrm{L}$ at location 2 (Table 4) which is far away from any pollution sources. While in Temsah Lake the maximum oxygen content of $10.20 \mathrm{mgO}_{2} / \mathrm{L}$ was recorded at location 9 and the minimum concentration $\left(5.20 \mathrm{mgO}_{2} / \mathrm{L}\right)$ at location 6 , probably attributed to the effect of acute pollution taking place through the disposal of untreated sewage and agricultural effluents from the sweet canal drains into the Lake.

Table 4: Hydrographic parameters of Bitter and Temsah Lakes during spring 2010.

\begin{tabular}{|c|c|c|c|c|c|c|c|}
\hline & Locations & Temp. & $\mathrm{pH}$ & $\mathrm{S} \% 0$ & DO & BOD & OOM \\
\hline \multirow{5}{*}{ 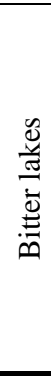 } & 1 & $\begin{array}{c}19.60-23.30 \\
(21.33)\end{array}$ & $\begin{array}{c}8.08-8.23 \\
(8.16)\end{array}$ & $\begin{array}{c}40.30-41.10 \\
(40.70)\end{array}$ & $\begin{array}{c}8.20-10.20 \\
(9.20)\end{array}$ & $\begin{array}{c}1.54-1.74 \\
(1.62)\end{array}$ & $\begin{array}{c}0.65-0.92 \\
(0.76)\end{array}$ \\
\hline & 2 & $\begin{array}{c}19.80-23.00 \\
(21.36)\end{array}$ & $\begin{array}{c}8.03-8.18 \\
(8.10)\end{array}$ & $\begin{array}{c}40.30-40.90 \\
(40.60)\end{array}$ & $\begin{array}{c}8.20-10.55 \\
(9.38)\end{array}$ & $\begin{array}{c}1.45-1.67 \\
(1.53)\end{array}$ & $\begin{array}{c}0.64-0.89 \\
(0.73) \\
\end{array}$ \\
\hline & 3 & $\begin{array}{c}21.15-26.00 \\
(22.90)\end{array}$ & $\begin{array}{c}8.04-8.20 \\
(8.10) \\
\end{array}$ & $\begin{array}{c}40.50-41.30 \\
(40.90)\end{array}$ & $\begin{array}{c}7.03-7.48 \\
(7.26) \\
\end{array}$ & $\begin{array}{c}1.53-2.05 \\
(1.61) \\
\end{array}$ & $\begin{array}{c}0.64-1.19 \\
(0.93) \\
\end{array}$ \\
\hline & 4 & $\begin{array}{c}21.25-27.45 \\
(23.43)\end{array}$ & $\begin{array}{c}8.05-8.27 \\
(8.13)\end{array}$ & $\begin{array}{c}40.20-40.30 \\
(40.25)\end{array}$ & $\begin{array}{c}7.17-7.91 \\
(7.54)\end{array}$ & $\begin{array}{c}1.38-1.92 \\
(1.75)\end{array}$ & $\begin{array}{c}0.56-1.55 \\
(1.19)\end{array}$ \\
\hline & 5 & $\begin{array}{c}21.20-25.80 \\
(23.06) \\
\end{array}$ & $\begin{array}{c}8.06-8.21 \\
(8.12) \\
\end{array}$ & $\begin{array}{c}40.60-41.20 \\
(40.90) \\
\end{array}$ & $\begin{array}{c}7.22-7.84 \\
(7.53) \\
\end{array}$ & $\begin{array}{c}1.37-1.85 \\
(1.61) \\
\end{array}$ & $\begin{array}{c}0.72-1.63 \\
(1.26) \\
\end{array}$ \\
\hline \multirow{4}{*}{ 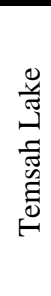 } & 6 & $\begin{array}{c}21.50-26.40 \\
(23.56)\end{array}$ & $\begin{array}{c}8.04-8.14 \\
(8.07)\end{array}$ & $\begin{array}{c}19.50-39.40 \\
(27.93)\end{array}$ & $\begin{array}{c}7.73-9.35 \\
(8.54) \\
\end{array}$ & $\begin{array}{c}3.21-3.65 \\
(3.42)\end{array}$ & $\begin{array}{c}3.04-3.25 \\
(3.09)\end{array}$ \\
\hline & 7 & $\begin{array}{c}21.20-26.40 \\
(23.26)\end{array}$ & $\begin{array}{c}8.11-8.14 \\
(8.12)\end{array}$ & $\begin{array}{c}22.40-32.20 \\
(27.30)\end{array}$ & $\begin{array}{c}8.40-9.24 \\
(8.82)\end{array}$ & $\begin{array}{c}3.15-3.45 \\
(3.25)\end{array}$ & $\begin{array}{c}2.40-2.79 \\
(2.53)\end{array}$ \\
\hline & 8 & $\begin{array}{c}21.10-25.30 \\
(22.83)\end{array}$ & $\begin{array}{c}8.13-8.21 \\
(8.16)\end{array}$ & $\begin{array}{c}29.50-37.80 \\
(33.65)\end{array}$ & $\begin{array}{c}8.03-10.10 \\
(9.07)\end{array}$ & $\begin{array}{c}3.24-3.39 \\
(6.60)\end{array}$ & $\begin{array}{c}1.06-2.13 \\
(1.39)\end{array}$ \\
\hline & 9 & $\begin{array}{c}21.00-27.50 \\
(23.66)\end{array}$ & $\begin{array}{c}8.02-8.22 \\
(8.13)\end{array}$ & $\begin{array}{c}28.70-30.80 \\
(29.75)\end{array}$ & $\begin{array}{c}8.04-10.20 \\
(9.12)\end{array}$ & $\begin{array}{c}3.19-3.35 \\
(3.21)\end{array}$ & $\begin{array}{c}1.92-2.02 \\
(1.51)\end{array}$ \\
\hline
\end{tabular}

Seasonally, the results (Table 5) indicated that, the dissolved oxygen values increased during spring and winter, while they decreased during summer because of high temperature which causes decreasing in the degree of solubility of atmospheric oxygen gas and due to the increase in oxygen consumption during decomposition of organic matters and oxidation of chemicals from effluents of different sources (Hamed, 2003).

Table 5: Seasonal variation of hydrochemical parameters in Bitter and Temsah lakes

\begin{tabular}{|c|c|c|c|c|c|c|c|}
\hline 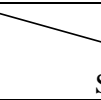 & neter & Temp. ${ }^{\circ} \mathrm{C}$ & $\mathrm{pH}$ & $\mathrm{S} \% 0$ & Do & BOD & OOM \\
\hline \multirow{4}{*}{ 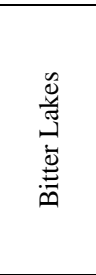 } & Autumn & $\begin{array}{c}24.10-30.60 \\
(26.74)\end{array}$ & $\begin{array}{c}7.91-8.12 \\
(8.04)\end{array}$ & $\begin{array}{c}39.30-41.20 \\
(40.21)\end{array}$ & $\begin{array}{c}5.95-7.69 \\
(6.76)\end{array}$ & $\begin{array}{c}1.21-2.82 \\
(1.74)\end{array}$ & $\begin{array}{c}0.72-1.93 \\
(1.24)\end{array}$ \\
\hline & Winter & $\begin{array}{c}16.90-19.25 \\
(18.35)\end{array}$ & $\begin{array}{c}8.05-8.29 \\
(8.16)\end{array}$ & $\begin{array}{c}39.20-40.90 \\
(40.03)\end{array}$ & $\begin{array}{c}6.30-9.12 \\
(7.40)\end{array}$ & $\begin{array}{c}1.28-2.15 \\
(1.71)\end{array}$ & $\begin{array}{c}0.76-2.06 \\
(1.38)\end{array}$ \\
\hline & Spring & $\begin{array}{c}19.60-27.45 \\
(22.86)\end{array}$ & $\begin{array}{c}8.03-8.27 \\
(8.14)\end{array}$ & $\begin{array}{c}40.20-41.30 \\
(40.67)\end{array}$ & $\begin{array}{c}7.03-10.55 \\
(8.18)\end{array}$ & $\begin{array}{c}1.37-2.05 \\
(1.65)\end{array}$ & $\begin{array}{c}0.56-1.63 \\
(0.94)\end{array}$ \\
\hline & Summer & $\begin{array}{c}28.75-31.35 \\
(30.16)\end{array}$ & $\begin{array}{c}7.95-8.11 \\
(8.02)\end{array}$ & $\begin{array}{c}39.90-41.60 \\
(40.50)\end{array}$ & $\begin{array}{c}5.70-7.70 \\
(6.69)\end{array}$ & $\begin{array}{c}1.19-2.59 \\
(1.87)\end{array}$ & $\begin{array}{c}0.96-2.16 \\
(1.63)\end{array}$ \\
\hline $\begin{array}{c}\text { Annual } \\
\text { mean }\end{array}$ & & $\begin{array}{c}22.34-27.16 \\
(24.53)\end{array}$ & $\begin{array}{c}7.99-8.19 \\
(8.09)\end{array}$ & $\begin{array}{c}39.65-41.25 \\
(40.40)\end{array}$ & $\begin{array}{c}6.25-8.77 \\
(7.26)\end{array}$ & $\begin{array}{c}1.26-2.40 \\
(1.74)\end{array}$ & $\begin{array}{c}0.75-1.95 \\
(1.29)\end{array}$ \\
\hline \multirow{4}{*}{ 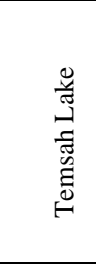 } & Autumn & $\begin{array}{c}24.50-29.60 \\
(27.06)\end{array}$ & $\begin{array}{c}7.78-8.29 \\
(8.11)\end{array}$ & $\begin{array}{c}24.70-39.10 \\
(31.43)\end{array}$ & $\begin{array}{c}5.44-6.71 \\
(6.15)\end{array}$ & $\begin{array}{c}2.12-5.85 \\
(3.58)\end{array}$ & $\begin{array}{c}1.62-4.16 \\
(2.83)\end{array}$ \\
\hline & Winter & $\begin{array}{c}16.20-19.80 \\
(18.15)\end{array}$ & $\begin{array}{c}7.86-8.28 \\
(8.09)\end{array}$ & $\begin{array}{c}7.30-39.10 \\
(18.56)\end{array}$ & $\begin{array}{c}5.87-7.33 \\
(6.45)\end{array}$ & $\begin{array}{c}2.12-3.88 \\
(2.83)\end{array}$ & $\begin{array}{c}1.71-4.14 \\
(2.72)\end{array}$ \\
\hline & Spring & $\begin{array}{c}21.00-27.50 \\
(23.80)\end{array}$ & $\begin{array}{c}8.02-8.22 \\
(8.13)\end{array}$ & $\begin{array}{c}19.50-39.40 \\
(30.04\end{array}$ & $\begin{array}{c}7.73-10.20 \\
(8.89)\end{array}$ & $\begin{array}{c}3.15-3.65 \\
\quad(3.33)\end{array}$ & $\begin{array}{c}1.06-3.25 \\
(2.33)\end{array}$ \\
\hline & Summer & $\begin{array}{c}29.50-32.50 \\
(31.25)\end{array}$ & $\begin{array}{c}7.89-8.31 \\
(8.09)\end{array}$ & $\begin{array}{c}30.60-40.10 \\
(35.31)\end{array}$ & $\begin{array}{c}5.20-7.48 \\
(6.36)\end{array}$ & $\begin{array}{c}1.89-5.92 \\
(3.67)\end{array}$ & $\begin{array}{c}1.91-4.24 \\
(2.93)\end{array}$ \\
\hline $\begin{array}{c}\text { Annual } \\
\text { mean }\end{array}$ & & $\begin{array}{c}22.80-27.35 \\
(25.07)\end{array}$ & $\begin{array}{c}7.89-8.28 \\
(8.11)\end{array}$ & $\begin{array}{c}20.53-39.43 \\
(28.84)\end{array}$ & $\begin{array}{c}6.06-7.93 \\
(6.96)\end{array}$ & $\begin{array}{c}2.32-4.83 \\
(3.35)\end{array}$ & $\begin{array}{c}1.58-3.95 \\
(2.70)\end{array}$ \\
\hline
\end{tabular}


On a seasonal scale, high oxygen values were recorded during winter due to the active water mixing and gas exchange, while slight increase was during spring associated with highest flourishing of phytoplankton in this season. On the other hand, the lowest value was recorded during summer and this may be due to the relatively high temperature and low density of phytoplankton. This illustrates that, the oxygen concentration is affected by the temperature pattern.

It was clear that BOD value increases gradually during winter and autumn and reaches its maximum during summer. This may be attributed to the increasing of microorganisms (bacteria) which needs DO for oxidizing the organic matter (Hamed, 2003). Water is considered fairly pure with a BOD of $3 \mathrm{ppm}$ and of doubtful purity when the BOD value reaches $5 \mathrm{ppm}$ (Anon, 1975). The BOD of the Lake water is slightly high indicating the presence of perceptible load of sewage continuously discharged into the area of investigation.

In general, the annual mean of BOD and OOM in Bitter Lakes was 1.74 and $1.29 \mathrm{mgO}_{2} / \mathrm{L}$, while in Temsah Lake the corresponding values were $3.35 \mathrm{mgO}_{2} / \mathrm{L}$ and $2.70 \mathrm{mgO}_{2} / \mathrm{L}$, respectively. The relatively high values of BOD and OOM in Temsah Lake may be due to the disposal of untreated sewage.

\section{Nutrient salts:}

The most important forms of nutrients in seawater of the inorganic nitrogen are ammonia, nitrite and nitrate. The concentrations of these nutrients usually lie in the range of $0.15-3 \mathrm{mgNH}_{3}-\mathrm{N} / \mathrm{L}, \quad 0.01-3 \mathrm{mgNO}_{2}-\mathrm{N} / \mathrm{L}$ and $0.1-35 \mathrm{mgNO}_{3}-\mathrm{N} / \mathrm{L}$ in oxygenated water (Riley and Chester, 1971).

Ammonia concentration in Bitter Lakes fluctuated between $0.19 \mu \mathrm{mol} / \mathrm{L}$ at location 2 and $5.76 \mu \mathrm{mol} / \mathrm{L}$ at location 4 , probably due to human activity at Fayed city, On the other hand, in Temsah Lake the lowest concentration of ammonia $(0.55$ $\mu \mathrm{mol} / \mathrm{L}$ ) was recorded at location 9 (Table 6), which is far away from any sources of pollution, while the highest concentration of ammonia $(9.79 \mu \mathrm{mol} / \mathrm{L})$ was recorded at location 6 (Table 7); this may be attributed to the high load of sewage from the effluents in this location.

Table 6: Nutrient Salts Concentration in Bitter and Temsah lakes during summer 2009.

\begin{tabular}{|c|c|c|c|c|c|c|}
\hline & Locations & $\begin{array}{c}\text { Ammonia } \\
\left(\mathrm{NH}_{4}\right) \mu \mathrm{mol} / \mathrm{L}\end{array}$ & $\begin{array}{c}\text { Nitrite } \\
\left(\mathrm{NO}_{2}\right) \mu \mathrm{mol} / \mathrm{L}\end{array}$ & $\begin{array}{c}\text { Nitrate } \\
\left(\mathrm{NO}_{3}\right) \mu \mathrm{mol} / \mathrm{L}\end{array}$ & $\begin{array}{c}\text { Phosphate } \\
\left(\mathrm{PO}_{4}\right) \mu \mathrm{mol} / \mathrm{L}\end{array}$ & $\begin{array}{c}\text { Silicate } \\
\left(\mathrm{SiO}_{3}\right) \mu \mathrm{mol} / \mathrm{L}\end{array}$ \\
\hline \multirow{5}{*}{ 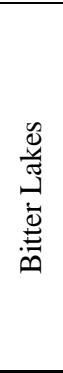 } & 1 & $\begin{array}{c}0.43-1.99 \\
(1.21)\end{array}$ & $\begin{array}{c}0.16-0.93 \\
(0.55)\end{array}$ & $\begin{array}{c}0.93-2.06 \\
(1.49)\end{array}$ & $\begin{array}{c}0.14-0.30 \\
(0.22)\end{array}$ & $\begin{array}{c}1.12-2.52 \\
(1.82)\end{array}$ \\
\hline & 2 & $\begin{array}{c}0.49-1.15 \\
(0.82)\end{array}$ & $\begin{array}{c}0.15-0.78 \\
(0.47)\end{array}$ & $\begin{array}{c}0.67-2.69 \\
(1.68)\end{array}$ & $\begin{array}{c}0.10-0.23 \\
(0.17)\end{array}$ & $\begin{array}{c}0.90-1.90 \\
(1.40)\end{array}$ \\
\hline & 3 & $\begin{array}{c}0.25-0.43 \\
(0.34)\end{array}$ & $\begin{array}{c}0.16-0.66 \\
(0.41)\end{array}$ & $\begin{array}{c}0.83-2.59 \\
(1.71)\end{array}$ & $\begin{array}{c}0.09-0.27 \\
(0.18)\end{array}$ & $\begin{array}{c}2.72-4.52 \\
(3.62)\end{array}$ \\
\hline & 4 & $\begin{array}{c}0.89-1.57 \\
(1.23) \\
\end{array}$ & $\begin{array}{c}0.18-0.73 \\
(0.46) \\
\end{array}$ & $\begin{array}{c}0.68-2.84 \\
(1.76) \\
\end{array}$ & $\begin{array}{c}0.07-0.48 \\
(0.28) \\
\end{array}$ & $\begin{array}{c}4.30-4.35 \\
(4.33) \\
\end{array}$ \\
\hline & 5 & $\begin{array}{c}0.55-1.05 \\
(0.80) \\
\end{array}$ & $\begin{array}{c}0.19-0.72 \\
(0.45) \\
\end{array}$ & $\begin{array}{c}0.75-2.35 \\
(1.55) \\
\end{array}$ & $\begin{array}{c}0.14-0.27 \\
(0.21) \\
\end{array}$ & $\begin{array}{c}4.80-4.95 \\
(4.88) \\
\end{array}$ \\
\hline \multirow{4}{*}{ 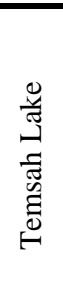 } & 6 & $\begin{array}{c}2.03-3.77 \\
(2.90)\end{array}$ & $\begin{array}{c}0.46-1.42 \\
(0.94)\end{array}$ & $\begin{array}{c}4.39-6.27 \\
(5.33)\end{array}$ & $\begin{array}{c}0.60-1.72 \\
(1.16)\end{array}$ & $\begin{array}{c}5.49-7.65 \\
(6.57)\end{array}$ \\
\hline & 7 & $\begin{array}{c}0.84-2.72 \\
(1.78)\end{array}$ & $\begin{array}{c}0.90-1.45 \\
(1.18) \\
\end{array}$ & $\begin{array}{c}2.88-4.45 \\
(3.67) \\
\end{array}$ & $\begin{array}{c}0.12-1.31 \\
(0.72) \\
\end{array}$ & $\begin{array}{c}5.40-6.90 \\
(6.15) \\
\end{array}$ \\
\hline & 8 & $\begin{array}{c}0.83-1.49 \\
(1.16)\end{array}$ & $\begin{array}{c}0.62-1.07 \\
(0.85)\end{array}$ & $\begin{array}{c}2.45-4.45 \\
(3.45)\end{array}$ & $\begin{array}{c}0.09-0.76 \\
(0.52)\end{array}$ & $\begin{array}{c}6.05-7.07 \\
(6.56)\end{array}$ \\
\hline & 9 & $\begin{array}{c}0.55-1.23 \\
(0.89)\end{array}$ & $\begin{array}{c}0.67-1.17 \\
(0.92)\end{array}$ & $\begin{array}{c}2.04-4.44 \\
(3.24)\end{array}$ & $\begin{array}{c}0.17-1.09 \\
(0.72)\end{array}$ & $\begin{array}{c}6.17-7.67 \\
(6.92)\end{array}$ \\
\hline
\end{tabular}


Table 7: Nutrient Salts Concentration in Bitter and Temsah lakes during winter 2010.

\begin{tabular}{|c|c|c|c|c|c|c|}
\hline & Locations & $\begin{array}{c}\text { Ammonia } \\
\left(\mathrm{NH}_{4}\right) \mu \mathrm{mol} / \mathrm{L}\end{array}$ & $\begin{array}{c}\text { Nitrite } \\
\left(\mathrm{NO}_{2}\right) \mu \mathrm{mol} / \mathrm{L}\end{array}$ & $\begin{array}{c}\text { Nitrate } \\
\left(\mathrm{NO}_{3}\right) \mu \mathrm{mol} / \mathrm{L}\end{array}$ & $\begin{array}{c}\text { Phosphate } \\
\left(\mathrm{PO}_{4}\right) \mu \mathrm{mol} / \mathrm{L}\end{array}$ & $\begin{array}{c}\text { Silicate } \\
\left(\mathrm{SiO}_{3}\right) \mu \mathrm{mol} / \mathrm{L}\end{array}$ \\
\hline \multirow{5}{*}{ 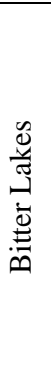 } & 1 & $\begin{array}{c}0.71-3.98 \\
(2.34)\end{array}$ & $\begin{array}{c}0.50-0.96 \\
(0.73)\end{array}$ & $\begin{array}{c}3.55-6.13 \\
(4.84)\end{array}$ & $\begin{array}{c}0.82-1.46 \\
(1.14)\end{array}$ & $\begin{array}{c}1.00-4.82 \\
(2.53)\end{array}$ \\
\hline & 2 & $\begin{array}{c}0.83-3.59 \\
(2.21)\end{array}$ & $\begin{array}{c}0.27-0.81 \\
(0.54)\end{array}$ & $\begin{array}{c}1.55-3.30 \\
(2.43)\end{array}$ & $\begin{array}{c}0.76-0.95 \\
(0.85)\end{array}$ & $\begin{array}{c}0.80-4.80 \\
(2.47)\end{array}$ \\
\hline & 3 & $\begin{array}{c}2.11-5.31 \\
(3.71)\end{array}$ & $\begin{array}{c}0.30-0.90 \\
(0.60)\end{array}$ & $\begin{array}{c}1.03-2.05 \\
(1.54) \\
\end{array}$ & $\begin{array}{c}0.62-0.99 \\
(0.81) \\
\end{array}$ & $\begin{array}{c}0.70-5.02 \\
(2.86) \\
\end{array}$ \\
\hline & 4 & $\begin{array}{c}1.86-5.76 \\
(3.81)\end{array}$ & $\begin{array}{c}0.30-0.49 \\
(0.39)\end{array}$ & $\begin{array}{c}3.84-7.61 \\
(5.73)\end{array}$ & $\begin{array}{c}0.68-0.73 \\
(0.70)\end{array}$ & $\begin{array}{c}0.35-5.60 \\
(2.98)\end{array}$ \\
\hline & 5 & $\begin{array}{c}1.05-1.33 \\
(1.19) \\
\end{array}$ & $\begin{array}{c}0.26-0.85 \\
(0.56) \\
\end{array}$ & $\begin{array}{c}2.47-5.11 \\
(3.79) \\
\end{array}$ & $\begin{array}{c}0.57-0.67 \\
(0.62) \\
\end{array}$ & $\begin{array}{c}1.30-3.50 \\
(2.40) \\
\end{array}$ \\
\hline \multirow{4}{*}{ 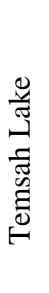 } & 6 & $\begin{array}{c}9.08-9.79 \\
(9.44)\end{array}$ & $\begin{array}{c}1.05-1.79 \\
(1.42)\end{array}$ & $\begin{array}{c}13.22-14.79 \\
(14.01)\end{array}$ & $\begin{array}{c}1.41-2.02 \\
(1.72)\end{array}$ & $\begin{array}{c}5.65-6.55 \\
(6.10)\end{array}$ \\
\hline & 7 & $\begin{array}{c}2.67-7.83 \\
(5.25) \\
\end{array}$ & $\begin{array}{c}0.15-1.05 \\
(0.60) \\
\end{array}$ & $\begin{array}{c}4.27-5.47 \\
(4.87) \\
\end{array}$ & $\begin{array}{c}1.18-1.46 \\
(1.32) \\
\end{array}$ & $\begin{array}{c}4.67-5.70 \\
(5.19) \\
\end{array}$ \\
\hline & 8 & $\begin{array}{c}4.66-4.74 \\
(6.20)\end{array}$ & $\begin{array}{c}0.17-1.13 \\
(0.65)\end{array}$ & $\begin{array}{c}3.78-4.17 \\
(3.98)\end{array}$ & $\begin{array}{c}1.01-1.38 \\
(1.20)\end{array}$ & $\begin{array}{c}3.35-4.40 \\
(3.88)\end{array}$ \\
\hline & 9 & $\begin{array}{c}3.91-4.04 \\
(3.98)\end{array}$ & $\begin{array}{c}0.77-1.00 \\
(0.89)\end{array}$ & $\begin{array}{c}4.11-5.11 \\
(4.61)\end{array}$ & $\begin{array}{c}1.06-1.32 \\
(1.19)\end{array}$ & $\begin{array}{c}2.35-4.50 \\
(3.43)\end{array}$ \\
\hline
\end{tabular}

Ammonia is biologically active compound present in most water as normal biological degradation product of nitrogenous organic matter. It reaches ground surface water through discharge of industrial waters containing ammonia as a byproduct or wastes from different sources of human activities (Train, 1978).

Nitrite concentration in Bitter Lakes varied between $0.15 \mu \mathrm{mol} / \mathrm{L}$ at location 2 during summer and $0.97 \mu \mathrm{mol} / \mathrm{L}$ at location 4 during spring. While the absolute values of nitrate concentration in Bitter Lakes fluctuated between $0.67 \mu \mathrm{mol} / \mathrm{L}$ at location 2 during summer and $7.61 \mu \mathrm{mol} / \mathrm{L}$ at location 4 during winter (Table 7). This may be due to increase in plankton biomass during summer that feed on such nutrients and cause depletion in its concentration. On the other hand, nitrite concentration in Temsah Lake recorded the minimum concentration $(0.12 \mu \mathrm{mol} / \mathrm{L})$ at location 9 (Table 8$)$, where there is no source of pollution and the maximum concentration $(1.79 \mu \mathrm{mol} / \mathrm{L})$ was recorded at location 6 . While the average values of nitrate concentrations in Temsah Lake fluctuated between $2.04 \mu \mathrm{mol} / \mathrm{L}$ at location 9 and $14.79 \mu \mathrm{mol} / \mathrm{L}$ at location 6 .

Table 8: Nutrient Salts Concentration in Bitter and Temsah lakes during autumn 2009.

\begin{tabular}{|c|c|c|c|c|c|c|}
\hline & Locations & $\begin{array}{c}\text { Ammonia } \\
\left(\mathrm{NH}_{4}\right) \mu \mathrm{mol} / \mathrm{L}\end{array}$ & $\begin{array}{c}\text { Nitrite } \\
\left(\mathrm{NO}_{2}\right) \mu \mathrm{mol} / \mathrm{L}\end{array}$ & $\begin{array}{c}\text { Nitrate } \\
\left(\mathrm{NO}_{3}\right) \mu \mathrm{mol} / \mathrm{L}\end{array}$ & $\begin{array}{c}\text { Phosphate } \\
\left(\mathrm{PO}_{4}\right) \mu \mathrm{mol} / \mathrm{L}\end{array}$ & $\begin{array}{c}\text { Silicate } \\
\left(\mathrm{SiO}_{3}\right) \mu \mathrm{mol} / \mathrm{L}\end{array}$ \\
\hline \multirow{5}{*}{ 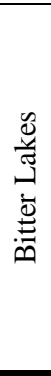 } & 1 & $\begin{array}{c}1.89-2.47 \\
(2.18)\end{array}$ & $\begin{array}{c}0.19-0.39 \\
(0.27)\end{array}$ & $\begin{array}{c}1.09-6.00 \\
(3.55)\end{array}$ & $\begin{array}{c}0.68-0.98 \\
(0.83)\end{array}$ & $\begin{array}{c}1.12-4.85 \\
(2.59)\end{array}$ \\
\hline & 2 & $\begin{array}{c}1.67-2.67 \\
(2.18) \\
\end{array}$ & $\begin{array}{c}0.28-0.52 \\
(0.40) \\
\end{array}$ & $\begin{array}{c}1.17-5.89 \\
(3.53) \\
\end{array}$ & $\begin{array}{c}0.68-0.83 \\
(0.76) \\
\end{array}$ & $\begin{array}{c}0.70-4.32 \\
(2.51) \\
\end{array}$ \\
\hline & 3 & $\begin{array}{c}0.90-1.45 \\
(1.18) \\
\end{array}$ & $\begin{array}{c}0.39-0.81 \\
(0.60) \\
\end{array}$ & $\begin{array}{c}1.16-6.40 \\
(3.94) \\
\end{array}$ & $\begin{array}{c}0.65-0.77 \\
(0.71) \\
\end{array}$ & $\begin{array}{c}0.42-4.40 \\
(2.41) \\
\end{array}$ \\
\hline & 4 & $\begin{array}{c}1.33-2.85 \\
(2.09) \\
\end{array}$ & $\begin{array}{c}0.34-0.90 \\
(0.62) \\
\end{array}$ & $\begin{array}{c}1.26-6.05 \\
(6.18)\end{array}$ & $\begin{array}{c}0.91-1.16 \\
(1.04)\end{array}$ & $\begin{array}{c}2.00-4.70 \\
(3.35) \\
\end{array}$ \\
\hline & 5 & $\begin{array}{c}0.90-1.08 \\
(0.99) \\
\end{array}$ & $\begin{array}{c}0.35-0.50 \\
(0.43) \\
\end{array}$ & $\begin{array}{c}1.27-6.35 \\
(5.84) \\
\end{array}$ & $\begin{array}{c}0.84-0.93 \\
(0.89) \\
\end{array}$ & $\begin{array}{c}0.22-4.90 \\
(2.56) \\
\end{array}$ \\
\hline \multirow{4}{*}{ 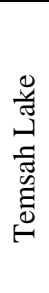 } & 6 & $\begin{array}{c}1.70-3.22 \\
(2.46) \\
\end{array}$ & $\begin{array}{c}0.44-1.48 \\
(0.96) \\
\end{array}$ & $\begin{array}{c}5.56-12.27 \\
(19.76) \\
\end{array}$ & $\begin{array}{c}0.79-1.51 \\
(1.15) \\
\end{array}$ & $\begin{array}{c}2.40-6.20 \\
(4.30)\end{array}$ \\
\hline & 7 & $\begin{array}{c}1.36-2.73 \\
(2.05) \\
\end{array}$ & $\begin{array}{c}0.34-1.64 \\
(0.99) \\
\end{array}$ & $\begin{array}{c}4.72-11.05 \\
(17.01)\end{array}$ & $\begin{array}{c}0.76-1.48 \\
(1.12) \\
\end{array}$ & $\begin{array}{c}3.85-4.90 \\
(4.38) \\
\end{array}$ \\
\hline & 8 & $\begin{array}{c}0.96-1.31 \\
(1.14) \\
\end{array}$ & $\begin{array}{c}0.13-1.10 \\
(0.62) \\
\end{array}$ & $\begin{array}{c}3.62-7.57 \\
(10.63) \\
\end{array}$ & $\begin{array}{c}0.65-1.19 \\
(0.92)\end{array}$ & $\begin{array}{c}2.10-5.40 \\
(3.75) \\
\end{array}$ \\
\hline & 9 & $\begin{array}{c}1.03-1.93 \\
(1.48)\end{array}$ & $\begin{array}{c}0.12-1.23 \\
(0.68)\end{array}$ & $\begin{array}{c}3.05-7.15 \\
\quad(9.12)\end{array}$ & $\begin{array}{c}0.65-1.27 \\
(0.96)\end{array}$ & $\begin{array}{c}1.05-3.62 \\
(2.34)\end{array}$ \\
\hline
\end{tabular}


The maximum value of DIP in Bitter Lakes was $1.46 \mu \mathrm{mol} / \mathrm{L}$ at location 1 during winter, while the minimum (0.07) was at location 4 during summer. In Temsah Lake, DIP values varied between $0.09 \mu \mathrm{mol} / \mathrm{L}$ at location 8 and $2.02 \mu \mathrm{mol} / \mathrm{L}$ at location 6.

Silicate concentration in Bitter Lakes fluctuated between $0.22 \mu \mathrm{mol} / \mathrm{L}$ during autumn at location 5 and $6.62 \mu \mathrm{mol} / \mathrm{L}$ during spring at location 4 . The importance of silicates lies in its significance for the construction of the cell wall of diatoms. This element is not a limiting factor for diatoms in the lake. In the same way at Temsah Lake, silicate concentrations differ from $1.05 \mu \mathrm{mol} / \mathrm{L}$ at location 9 which is far away from any source of pollution to $7.65 \mu \mathrm{mol} / \mathrm{L}$ at location 6 . William (1991) reported dissolved silica content between $0.03 \mathrm{mg} / \mathrm{L}$ and $0.20 \mathrm{mg} / \mathrm{L}$ as limiting level for diatom growth.

Seasonally, nutrient salts recorded the highest values during winter in the present study and this is may be due to tidal current of the water column, which mixes the bottom water with the surface area. These results agree with that of Saied and ElDeek (2004), while the lowest values were recorded during autumn and spring may be due to the production of phytoplankton standing crop that agree with Nassar and Hamed (2003) studies.

The annual and seasonal variations of nutrient salts viz: ammonia, nitrite, nitrate, phosphate and silicate in Bitter and Temsah Lakes are given in Table 10.

In general, concentrations of nutrient salts in Temsah Lake are relatively high compared with that of Bitter Lakes probably due to heavy load of sewage and agricultural effluents discharge into Temsah Lake.

Table 9: Nutrient Salts Concentration in Bitter and Temsah lakes during spring 2010.

\begin{tabular}{|c|c|c|c|c|c|c|}
\hline & Locations & $\begin{array}{c}\text { Ammonia } \\
(\mathrm{NH} 4) \mu \mathrm{mol} / \mathrm{L}\end{array}$ & $\begin{array}{c}\text { Nitrite } \\
\left(\mathrm{NO}_{2}\right) \mu \mathrm{mol} / \mathrm{L}\end{array}$ & $\begin{array}{c}\text { Nitrate } \\
\left(\mathrm{NO}_{3}\right) \mu \mathrm{mol} / \mathrm{L}\end{array}$ & $\begin{array}{c}\text { Phosphate } \\
\left(\mathrm{PO}_{4}\right) \mu \mathrm{mol} / \mathrm{L}\end{array}$ & $\begin{array}{c}\text { Silicate } \\
\left(\mathrm{SiO}_{3}\right) \mu \mathrm{mol} / \mathrm{L}\end{array}$ \\
\hline \multirow{5}{*}{ 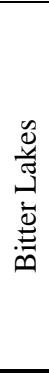 } & 1 & $\begin{array}{c}0.37-1.71 \\
(1.04)\end{array}$ & $\begin{array}{c}0.42-0.92 \\
(0.67)\end{array}$ & $\begin{array}{c}1.33-4.08 \\
(2.35)\end{array}$ & $\begin{array}{c}0.37-0.90 \\
(0.61)\end{array}$ & $\begin{array}{c}1.17-4.67 \\
(3.49)\end{array}$ \\
\hline & 2 & $\begin{array}{c}0.19-1.80 \\
(0.99)\end{array}$ & $\begin{array}{c}0.23-0.68 \\
(0.46)\end{array}$ & $\begin{array}{c}1.49-5.15 \\
(2.97)\end{array}$ & $\begin{array}{c}0.28-0.76 \\
(0.46)\end{array}$ & $\begin{array}{c}1.05-4.10 \\
(2.90)\end{array}$ \\
\hline & 3 & $\begin{array}{c}0.24-0.77 \\
(0.51)\end{array}$ & $\begin{array}{c}0.31-0.85 \\
(0.58)\end{array}$ & $\begin{array}{c}1.35-4.52 \\
(2.94)\end{array}$ & $\begin{array}{c}0.28-0.82 \\
(0.53)\end{array}$ & $\begin{array}{c}1.40-4.40 \\
(3.29)\end{array}$ \\
\hline & 4 & $\begin{array}{c}0.49-1.17 \\
(0.83)\end{array}$ & $\begin{array}{c}0.43-0.97 \\
(0.70)\end{array}$ & $\begin{array}{c}1.49-4.06 \\
(2.77)\end{array}$ & $\begin{array}{c}0.41-0.96 \\
(0.69)\end{array}$ & $\begin{array}{c}4.27-6.62 \\
(5.59)\end{array}$ \\
\hline & 5 & $\begin{array}{c}0.91-1.72 \\
(1.32) \\
\end{array}$ & $\begin{array}{c}0.25-0.67 \\
(0.46) \\
\end{array}$ & $\begin{array}{c}2.13-4.16 \\
(3.15) \\
\end{array}$ & $\begin{array}{c}0.39-0.76 \\
(0.57) \\
\end{array}$ & $\begin{array}{c}4.40-5.40 \\
(4.92) \\
\end{array}$ \\
\hline \multirow{4}{*}{ 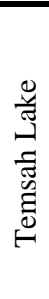 } & 6 & $\begin{array}{c}2.04-3.95 \\
(2.99)\end{array}$ & $\begin{array}{c}0.91-1.54 \\
(1.22)\end{array}$ & $\begin{array}{c}9.30-13.32 \\
(11.31)\end{array}$ & $\begin{array}{c}0.78-1.83 \\
(1.31)\end{array}$ & $\begin{array}{c}6.30-7.40 \\
(6.85)\end{array}$ \\
\hline & 7 & $\begin{array}{c}1.32-3.09 \\
(2.21)\end{array}$ & $\begin{array}{c}0.86-1.15 \\
(1.01)\end{array}$ & $\begin{array}{c}7.83-8.44 \\
(8.14)\end{array}$ & $\begin{array}{c}0.72-1.41 \\
(1.07)\end{array}$ & $\begin{array}{c}4.65-5.40 \\
(5.03)\end{array}$ \\
\hline & 8 & $\begin{array}{c}0.80-1.11 \\
(0.96)\end{array}$ & $\begin{array}{c}0.68-1.06 \\
(0.86)\end{array}$ & $\begin{array}{c}2.86-5.40 \\
(4.13)\end{array}$ & $\begin{array}{c}0.55-1.16 \\
(0.86)\end{array}$ & $\begin{array}{c}3.30-6.35 \\
(4.83)\end{array}$ \\
\hline & 9 & $\begin{array}{c}0.88-1.23 \\
(1.05) \\
\end{array}$ & $\begin{array}{c}0.70-1.13 \\
(0.92)\end{array}$ & $\begin{array}{c}3.88-4.79 \\
(4.34) \\
\end{array}$ & $\begin{array}{c}0.63-1.06 \\
(0.85) \\
\end{array}$ & $\begin{array}{c}2.62-4.75 \\
(3.69)\end{array}$ \\
\hline
\end{tabular}


Table 10: Seasonal variation of nutrient salts in Bitter and Temsah lakes

\begin{tabular}{|c|c|c|c|c|c|c|}
\hline \multicolumn{2}{|c|}{$\underbrace{\text { Parameter }}_{\text {Season }}$} & $\begin{array}{c}\text { Ammonia } \\
\left(\mathrm{NH}_{4}\right) \\
\mu \mathrm{mol} / \mathrm{L}\end{array}$ & $\begin{array}{c}\text { Nitrite } \\
\left(\mathrm{NO}_{2}\right) \mu \mathrm{mol} / \\
\mathrm{L}\end{array}$ & $\begin{array}{c}\text { Nitrate } \\
\left(\mathrm{NO}_{3}\right) \mu \mathrm{mol} / \\
\mathrm{L}\end{array}$ & $\begin{array}{c}\text { Phosphate } \\
\left(\mathrm{PO}_{4}\right) \\
\mu \mathrm{mol} / \mathrm{L}\end{array}$ & $\begin{array}{c}\text { Silicate } \\
\left(\mathrm{SiO}_{4}\right) \\
\mu \mathrm{mol} / \mathrm{L}\end{array}$ \\
\hline \multirow{4}{*}{ 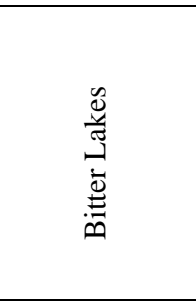 } & autumn & $\begin{array}{c}0.19-1.80 \\
(0.94)\end{array}$ & $\begin{array}{c}0.15-0.93 \\
(0.47)\end{array}$ & $\begin{array}{c}0.67-2.84 \\
(1.64)\end{array}$ & $\begin{array}{c}0.28-0.90 \\
(0.59)\end{array}$ & $\begin{array}{c}0.90-4.95 \\
(3.21)\end{array}$ \\
\hline & winter & $\begin{array}{c}0.71-5.76 \\
(3.24)\end{array}$ & $\begin{array}{c}0.19-0.90 \\
(0.47)\end{array}$ & $\begin{array}{c}1.33-5.15 \\
(2.98)\end{array}$ & $\begin{array}{c}0.07-0.48 \\
(0.21)\end{array}$ & $\begin{array}{c}0.22-5.60 \\
(2.83)\end{array}$ \\
\hline & Spring & $\begin{array}{c}0.25-1.99 \\
(0.88)\end{array}$ & $\begin{array}{c}0.23-0.97 \\
(0.57)\end{array}$ & $\begin{array}{c}1.09-6.40 \\
(3.36)\end{array}$ & $\begin{array}{c}0.65-1.18 \\
(0.84)\end{array}$ & $\begin{array}{c}1.05-6.62 \\
(3.75)\end{array}$ \\
\hline & summer & $\begin{array}{c}0.90-2.85 \\
(1.72)\end{array}$ & $\begin{array}{c}0.26-0.96 \\
(0.56)\end{array}$ & $\begin{array}{c}1.03-7.61 \\
(3.66)\end{array}$ & $\begin{array}{c}0.57-1.46 \\
(0.83) \\
\end{array}$ & $\begin{array}{c}0.42-4.85 \\
(2.73) \\
\end{array}$ \\
\hline Annual mean & & $\begin{array}{c}0.51-3.10 \\
(1.69) \\
\end{array}$ & $\begin{array}{c}0.21-0.94 \\
(0.52) \\
\end{array}$ & $\begin{array}{c}1.03-5.50 \\
(2.91) \\
\end{array}$ & $\begin{array}{c}0.39-1.01 \\
(0.62) \\
\end{array}$ & $\begin{array}{c}0.65-5.51 \\
(3.13) \\
\end{array}$ \\
\hline \multirow{4}{*}{ 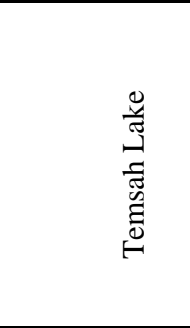 } & autumn & $\begin{array}{c}0.96-3.22 \\
(1.78) \\
\end{array}$ & $\begin{array}{c}0.15-1.79 \\
(0.89)\end{array}$ & $\begin{array}{c}3.78-14.79 \\
(6.87) \\
\end{array}$ & $\begin{array}{c}1.01-2.02 \\
(1.36) \\
\end{array}$ & $\begin{array}{c}2.10-6.20 \\
(3.96) \\
\end{array}$ \\
\hline & winter & $\begin{array}{c}2.67-9.79 \\
(5.84)\end{array}$ & $\begin{array}{c}0.12-1.64 \\
(0.81)\end{array}$ & $\begin{array}{c}2.86-13.32 \\
\quad(6.98)\end{array}$ & $\begin{array}{c}0.09-1.72 \\
(0.73)\end{array}$ & $\begin{array}{c}1.05-6.55 \\
(4.37)\end{array}$ \\
\hline & Spring & $\begin{array}{c}0.55-3.77 \\
(1.68)\end{array}$ & $\begin{array}{c}0.67-1.54 \\
(1.01)\end{array}$ & $\begin{array}{c}3.05-12.27 \\
(6.87)\end{array}$ & $\begin{array}{c}0.65-1.51 \\
\quad(1.04)\end{array}$ & $\begin{array}{c}2.62-7.40 \\
(5.09)\end{array}$ \\
\hline & Summer & $\begin{array}{c}0.80-3.95 \\
(1.80)\end{array}$ & $\begin{array}{c}0.46-1.45 \\
(0.97)\end{array}$ & $\begin{array}{c}2.04-6.27 \\
(3.92) \\
\end{array}$ & $\begin{array}{c}0.55-1.83 \\
(1.02)\end{array}$ & $\begin{array}{c}5.40-7.67 \\
(6.55) \\
\end{array}$ \\
\hline Annual mean & & $\begin{array}{c}1.25-5.18 \\
(2.78)\end{array}$ & $\begin{array}{c}0.35-1.61 \\
(0.92)\end{array}$ & $\begin{array}{c}2.93-11.66 \\
(6.16)\end{array}$ & $\begin{array}{c}0.58-1.77 \\
(1.03)\end{array}$ & $\begin{array}{c}2.79-5.46 \\
(4.99)\end{array}$ \\
\hline
\end{tabular}

In conclusion, the level of nutrient salts indicated that, Bitter and Temsah Lakes are in the eutrophic state according to the standard levels reported by Franco (1983) (Table 11).

Table 11: Comparison of annual mean values between the present study and previous studies.

\begin{tabular}{|c|c|c|c|c|c|c|c|c|c|c|c|c|}
\hline parameter & Temp & $\mathrm{pH}$ & S \%o & DO & BOD & OOM & $\mathrm{NH}_{4}$ & $\mathrm{NO}_{2}$ & $\mathrm{NO}_{3}$ & $\mathrm{PO}_{4}$ & $\mathrm{SiO}_{4}$ & \multirow[t]{2}{*}{ Reference } \\
\hline Area & & & & & & & & & & & & \\
\hline Bitter lakes & 24.53 & 8.09 & 40.40 & 7.26 & 1.74 & 1.29 & 1.59 & 0.52 & 2.91 & 0.62 & 3.13 & Present Study \\
\hline Temsah Lake & 25.07 & 8.11 & 28.84 & 6.96 & 3.35 & 2.7 & 2.78 & 0.92 & 6.16 & 1.03 & 4.99 & Present Study \\
\hline Temsah Lake & 24.13 & 8.08 & 32.04 & 4.46 & --- & ---- & ---- & ---- & ---- & --- & ---- & Mourad, 1996 \\
\hline Lake Manzala & 21.81 & 8.03 & 4.63 & 5.80 & 5.66 & 11.28 & 63.1 & 3.48 & 5.91 & 11.56 & 94.11 & Hamed, 2003 \\
\hline Lake Manzala & --- & --- & --- & --- & --- & --- & 128.45 & 5.81 & 9.25 & 24.6 & 142.5 & $\begin{array}{c}\text { Fahmy et al., } \\
1997\end{array}$ \\
\hline Suez Canal & --- & 8.22 & 38.57 & 6.23 & --- & --- & --- & --- & --- & --- & --- & $\begin{array}{c}\text { Abd El- } \\
\text { Rahman, } 1997\end{array}$ \\
\hline $\begin{array}{c}\begin{array}{c}\text { Oligotrophic } \\
\text { level }\end{array} \\
\text { Eutrophic level } \\
\end{array}$ & & & & & & & $\begin{array}{l}0.51 \\
2.02 \\
\end{array}$ & & $\begin{array}{l}0.51 \\
4.02 \\
\end{array}$ & $\begin{array}{l}0.05 \\
0.3 \\
\end{array}$ & & \\
\hline
\end{tabular}

Generally, the calculated ratios for N: P: Si (Table 12) were lower than that of Redfield ratios N: P: Si (16: 16: 1) (Redfield et al., 1963). Thus, nitrogen is the limiting factor for phytoplankton growth in the Bitter and Temsah Lakes. 
Table (12): Mean TIN/DRP, Si/DRP and TIN/Si ratios of Bitter and Temsah Lakes.

\begin{tabular}{|c|c|c|c|c|}
\hline \multicolumn{2}{|c|}{ Locations } & TIN/DRP & $\mathrm{Si} / \mathrm{DRP}$ & TIN/Si \\
\hline \multirow{6}{*}{ 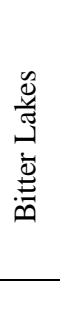 } & 1 & 7.619 & 3.751 & 2.031 \\
\hline & 2 & 9.180 & 4.078 & 2.251 \\
\hline & 3 & 7.092 & 4.711 & 1.505 \\
\hline & 4 & 9.705 & 5.972 & 1.625 \\
\hline & 5 & 8.382 & 6.448 & 1.300 \\
\hline & Mean area & 8.396 & 4.992 & 1.742 \\
\hline \multirow{5}{*}{ 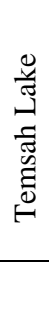 } & 6 & 12.283 & 4.466 & 2.750 \\
\hline & 7 & 10.086 & 4.911 & 2.053 \\
\hline & 8 & 8.823 & 5.543 & 1.592 \\
\hline & 9 & 8.073 & 4.477 & 1.803 \\
\hline & Mean area & 9.816 & 4.849 & 2.049 \\
\hline
\end{tabular}

The statistical calculations were carried out using statistical computer program. Correlation coefficients were calculated between all pairs of measured variables (Tables 13 and 14) and the results indicated that:

Table 13: correlation coefficient marked between pairs of measured variables in Bitter Lakes.

\begin{tabular}{|c|c|c|c|c|c|c|c|c|c|c|c|}
\hline & Temp. & $\mathrm{pH}$ & $\mathrm{S} \%$ D & $\mathrm{DO}$ & $\mathrm{BOD}$ & $\mathrm{OOM}$ & $\mathrm{NH}_{3}$ & $\mathrm{NO}_{2}$ & $\mathrm{NO}_{3}$ & $\mathrm{PO}_{4}$ & $\mathrm{SiO}_{4}$ \\
\hline Temp. & 1.00 & & & & & & & & & & \\
\hline $\mathrm{pH}$ & -0.10 & 1.00 & & & & & & & & & \\
\hline $\mathrm{S} \%$ co & 0.75 & -0.19 & 1.00 & & & & & & & & \\
\hline $\mathrm{DO}$ & -0.52 & 0.47 & 0.08 & 1.00 & & & & & & & \\
\hline $\mathrm{BOD}$ & 0.32 & -0.18 & -0.31 & -0.36 & 1.00 & & & & & & \\
\hline $\mathrm{OOM}$ & 0.30 & -0.39 & -0.33 & -0.43 & 0.46 & 1.00 & & & & & \\
\hline $\mathrm{NH}_{3}$ & -0.03 & -0.04 & -0.23 & -0.23 & 0.19 & -0.04 & 1.00 & & & & \\
\hline $\mathrm{NO}_{2}$ & -0.32 & 0.21 & -0.00 & 0.41 & -0.19 & 0.06 & 0.04 & 1.00 & & & \\
\hline $\mathrm{NO}_{3}$ & -0.56 & 0.60 & -0.03 & 0.38 & -0.07 & -0.06 & 0.02 & 0.27 & 1.00 & & \\
\hline $\mathrm{PO}_{4}$ & -0.56 & 0.43 & 0.18 & 0.53 & -0.15 & 0.01 & 0.48 & 0.54 & 0.47 & 1.00 & \\
\hline $\mathrm{SiO}_{4}$ & 0.13 & 0.21 & -0.31 & 0.19 & 0.19 & -0.03 & 0.24 & 0.09 & 0.44 & 0.38 & 1.00 \\
\hline
\end{tabular}

Table 14: correlation coefficient marked between pairs of measured variables in Temsah Lake.

\begin{tabular}{|c|c|c|c|c|c|c|c|c|c|c|c|}
\hline & Temp. & $\mathrm{pH}$ & $\mathrm{S} \%$ DO & $\mathrm{DO}$ & $\mathrm{BOD}$ & $\mathrm{OOM}$ & $\mathrm{NH}_{3}$ & $\mathrm{NO}_{2}$ & $\mathrm{NO}_{3}$ & $\mathrm{PO}_{4}$ & $\mathrm{SiO}_{4}$ \\
\hline Temp. & 1.00 & & & & & & & & & & \\
\hline $\mathrm{pH}$ & 0.05 & 1.00 & & & & & & & & & \\
\hline $\mathrm{S} \%$ co & 0.88 & 0.12 & 1.00 & & & & & & & & \\
\hline $\mathrm{DO}$ & -0.26 & 0.24 & 0.03 & 1.00 & & & & & & & \\
\hline $\mathrm{BOD}$ & 0.15 & -0.54 & 0.37 & -0.10 & 1.00 & & & & & & \\
\hline $\mathrm{OOM}$ & 0.09 & -0.74 & -0.02 & -0.55 & 0.25 & 1.00 & & & & & \\
\hline $\mathrm{NH}_{3}$ & 0.19 & -0.30 & 0.14 & -0.46 & 0.24 & 0.52 & 1.00 & & & & \\
\hline $\mathrm{NO}_{2}$ & 0.04 & -0.52 & 0.20 & 0.02 & 0.30 & 0.45 & 0.04 & 1.00 & & & \\
\hline $\mathrm{NO}_{3}$ & -0.32 & -0.36 & -0.06 & 0.52 & 0.30 & 0.32 & 0.11 & 0.61 & 1.00 & & \\
\hline $\mathrm{PO}_{4}$ & -0.55 & 0.70 & -0.58 & -0.11 & 0.17 & 0.50 & 0.20 & 0.50 & 0.36 & 1.00 & \\
\hline $\mathrm{SiO}_{4}$ & 0.57 & -0.48 & 0.46 & -0.06 & 0.34 & 0.33 & 0.04 & 0.43 & 0.19 & 0.09 & 1.00 \\
\hline
\end{tabular}

1) Temperature is correlated with salinity, where positive correlation $(r=0.75)$ in Bitter Lakes and $(r=0.88)$ in Temsah Lake. This is due to increasing of salinity during hot seasons and vice versa.

2) The negative correlation between temperature and DO ( $r=-0.52)$ in Bitter Lakes and $(r=-0.26)$ in Temsah Lake is due to the solubility product of DO decreasing with increasing temperature. 
3) The positive correlation $(r=0.43)$ in Bitter Lakes and $(r=0.70)$ in Temsah Lake obtained between the reactive phosphate and $\mathrm{pH}$ indicated that both two parameters are depending on the photosynthetic process.

4) The negative correlation between DO and BOD is $r=-0.36$ and OOM (-0.43) in Bitter Lakes, while in Temsah Lake correlation between DO and BOD $(r=-0.10)$ and OOM (-0.55) indicated that with increasing the rate of organic matter degradation either chemically or biologically the DO concentration decreases.

5) The highly significant positive correlation coefficient between BOD and OOM ( $r=$ 0.46) in Bitter Lakes and in Temsah Lake $(r=0.25)$ is probably due to organic matter degradation either chemically or biologically.

6) The high significant positive correlation coefficients between nutrient salts concentrations (Tables 13 and 14) are attributed to the conditions responsible for the enrichment of different nutrients are similar.

\section{REFERENCES}

Abdallah, A. M.; El-Samra, M. I. and Hamed M. H. (1994). Journal of Environmental Science, Mansoura University, Faculty of Science, 7: 11.

Abd El-Rahman, N. S. (1997). Suez Canal as a link in the migration of zooplankton between the Mediterranean and Red Seas. Ph. D. Thesis, Zoology Department, Faculty of Science, Suez Canal University, Ismailia, Egypt.

American Public Health Association, APHA (1995). Standard methods for the examination of wastewater, $19^{\text {th }}$ Ed. New York, 1015pp.

Anon, (1975): Environmental Control and Public Health, Water Analysis, Standards and Treatment, Eyre and Spottiswoode, Ltd., ECPH, 131 pp.

Ashour, S.; Hewedy, M.; Abd-Allah, S.; Gab-Allah, M. and Toullabah, H. (2000). The relationships between biotic and abiotic factors in the Great Bitter (El-Morra) Lakes. Journal of Microbiology and Viruses, 9(B): 195-209.

Badawy, A. (2001). Status of the crustal stress in Egypt as inferred from earthquake focal mechanisms and borehole breakouts. Tectonophysics, 343: 49-61

El-Bassat, RA. (2008). Composition and abundance of the zooplankton community in the Bitter Lakes, Egypt, in relation to environmental factors. African Journal of Aquatic Science, 33(3): 233-240.

El-Ibiary, M. G. (1981). Shallow subsurface geological and geophysical in Lake Temsah, Suez Canal area, Egypt. M. Sc. Thesis submitted to Geology Department, Faculty of Science, Tanta University, Egypt, 200pp.

El-Shazley, E. M.; Abdel Hady, M. A.; El-Ghawaby, M. A.; El-Kassas, I. A. and ElShazley, M. M. (1974). Geology of Sinai Peninsula from ERTS-1 satellite images. Remote sensing research project. Academy of Scientific Research and Technology, Cairo, Egypt.

ETPS, (1995). Environmental testing of pollution status in Lake Temsah, Abou Attwa water reuse center research and training, Ismailia, Egypt, $356 \mathrm{pp}$.

Fahmy, M. A.; Abbas, M. M. and Emara, H. I. (1997). Chemical studies on the impact of pollution in Lake Manzala, Egypt. Proceedings of the $7^{\text {th }}$ International Conference on Environmental Protection is a Must, 20-22 My, 1997, Alexandria, Egypt, Organized by NIOF (National Institute of Oceanography and Fisheries) and I. S. A. (International Scientists Association).

Franco, P. (1983). Fattori influent Sulla productivita dell Adriatica Settentrionale. Proc. Int. Cont. Problems of Adriatic Sea, Trieste pp. 155-174.

Hamed, M. A. (2003): Hydrochemistry and nutrients of Lake Manzala, Egypt. J. Egypt. Acad. Soc. Environ. Develop. (Environmental Studies), (2, P) 29-48.

Holmes, A. (1965). Principals of physical geology, 2nd edition. ELBS and Nelson, London, 190pp. 
Kaiser, M. F.; Amin, A. S. and Aboulela, H. A. (2008). Environmental hazards in the ElTemsah Lake, Suez Canal district, Egypt. Advances in Geosciences and Remote Sensing, pp. 57-70.

Mohamed, A. Hamed (2005). Assessment of heavy metals among suspended particulates and dissolved phases in Suez Canal water

Morcos, S. A., (1970). Physical and chemical oceanography of the Red Sea. Oceanogr. Mar. Biol. Ann. Rev., 8: 73-203

Morcos, S. A. and Riley, J. P. (1966). Chlorinity, Salinity, Density and Conductivity of seawater from the Suez Canal region. Deep- Suez Research, 13: 741-749.

Mourad, F. A. (1996). Heavy Metal pollution in Temsah Lake. M. Sc. Thesis, Marine Biology Department, Faculty of Science, Suez Canal Univ., Ismailia, Egypt

Nassar, M. Z. and Hamed, M. A. (2003). Phytoplankton sanding crop and species diversity in relation to some water characteristics of Suez Bay (Red Sea), Egypt. Egypt. J. Aquat. Biol. and Fish. 7 (3): 25-48.

Redfield, A. C.; Katchum, B. H. and Richards, S. A. (1963). The influence of organisms on the composition of seawater, In: the sea, vol. 2, M. N. Hill (Ed.) John Wily and Sons, New York, pp. 26- 67.

Riley, J. P. and Chester, R. (1971). Introduction to marine chemistry. Academic Press, London and New York, 465pp.

Saied, M. M. and El-Deek, M. S. (2004). Inorganic nitrogen, reactive phosphate and silicate in the seawater in front of Hurghada, Red Sea, Egypt. Bull. Nat. Inst. Oceanogr. \& Fish.

Strickland, J. D. H and Parsons, T. R. (1972). Practical handbook of seawater analysis. Bull. Fish. Res. Bd. Can. 167: 311pp.

Train, R. E. (1978). Quality Criteria from water. U. S. Environmental Protection Agency. Washington D. C. (USA).

William, E. (1991). Planktonic diatoms-ecological review. Algological studies, 62: 69-106.

Winkler, L. W. (1988). The determination of dissolved oxygen in water. Ber. Deut. Chem. Ges., 21: 2843-2848.

\section{ARABIC SUMMARY}

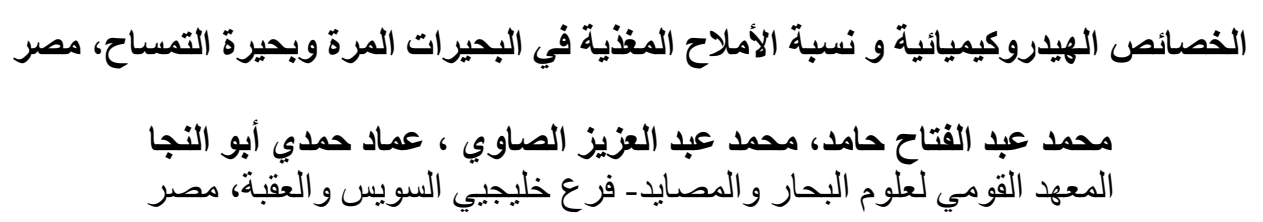

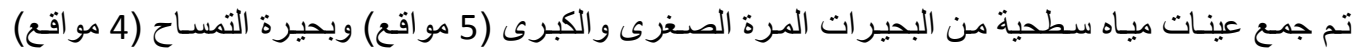

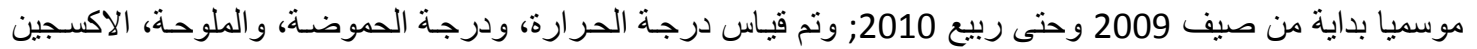

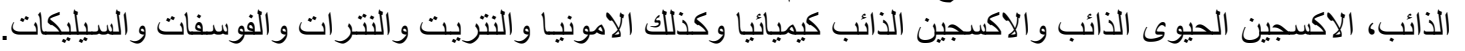

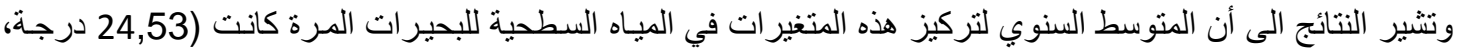

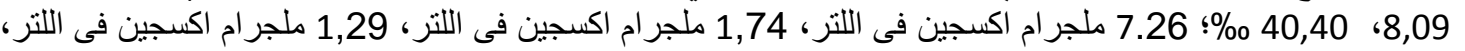

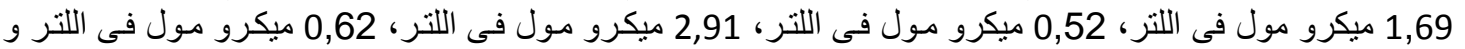

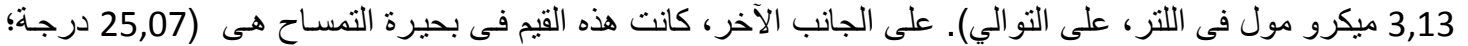

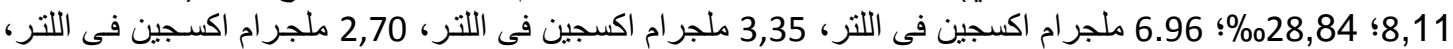

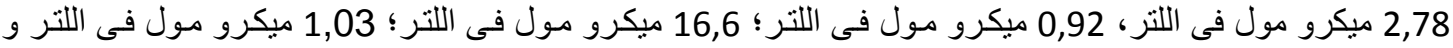

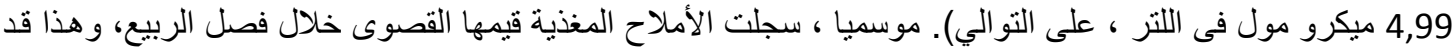

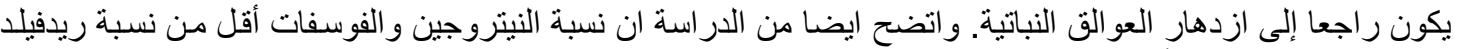

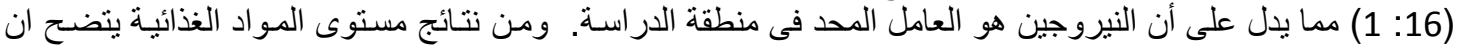

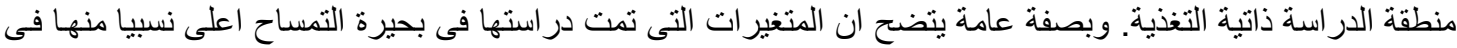

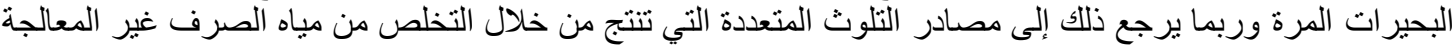
ومياه الصرف الزر اعي من ترعة المياه الحلوة الى البحيرات. 